\title{
Bidirectional DC/AC energy flow measurement
}

\author{
Pēteris Apse-Apsitis, Ansis Avotins, Leonids Ribickis \\ Institute of Industrial Electronics and Electrical Engineering \\ Riga Technical university \\ Riga, Latvia \\ peteris.apse-apsitis@rtu.lv, ansis.avotins@rtu.lv, leonids.ribickis@rtu.lv
}

\begin{abstract}
Bidirectional energy flow monitoring near $230 \mathrm{~V}$ AC or $600 \mathrm{~V}$ DC micro-grid consumers - industrial devices or household consumers - rise questions regarding measurement methods and devices self power consumption, price and precision. Article deals with overall $3 \times 230 \mathrm{~V}+\mathrm{N}$ AC and $600 \mathrm{~V}$ DC micro-grid bidirectional monitoring system design as well as measurement modules communication and data logging.
\end{abstract}

Keywords-power conversion, AC/DC micro-grids, bidirectional power flow, energy measurement,

I.

\section{INTRODUCTION}

A lot of electrical energy consumers or sources operate on $\mathrm{DC}$ and contain $\mathrm{AC} / \mathrm{DC}$ or $\mathrm{DC} / \mathrm{AC}$ converters thus making them possible to use them in $\mathrm{AC}$ environment.

DC energy storage devices like super capacitors, batteries, hydrogen cells etc. allow to store and re-use energy, thus making overall system more effective from energy utilisation point of view. Such devices are connected to the DC micro-grid via bidirectional converters.

Active front-end AC-DC-AC convertor module is used to feed DC micro-grid and return excess of energy in to AC grid in case if isn't possible to store energy in to storage devices.

Simultaneous energy flow monitoring near DC micro-grid consumers/sources and AC grid allow to determine system efficiency, as well as weak system points from energy flow point of view. Mentioned above allow to make changes in to devices workflow in order to increase efficiency, if possible and necessary.

Article deals with overall monitoring system design as well as measurement modules communication and data logging. Several details cannot be represented here due to current Project restrictions.

\section{SugGestions On EleCtrical ENERGy MEASUREMENT}

Typically energy consumption calculations are based on instant power values, especially if consumer generate nonsinusoidal current form. Instant current and voltage value readings (samples) are made and following multiplication are used to calculate instant power, average power or consumed energy [1], [2]. Sampling rate must be at least $4,2 \mathrm{KHz}$ or 42 samples per 1/2T according to EN 61000-3-2 [7], and Nyquist frequency.

Other method are voltage and current values averaging via multi-order delta-sigma modulation and the following multiplication [3].
Thus electrical energy measuring/monitoring device installation near every consumer or generator are expensive. Several methods are proposed to lower costs, for example [4], [5], in order to achieve widespread electrical energy measuring/ monitoring devices installations. The main disadvantage is necessity of separate low power source for measuring IC's power feed and resulting increase in to measuring device selfconsumption.

Between AC and DC energy measurements are a difference due to $\mathrm{AC}$ and $\mathrm{DC}$ environment difference, especially if bidirectional energy flow take place.

Non-even sampling energy consumption measuring method was proposed to overcome mentioned above disadvantages [6]. Method allow to measure bi-directional AC or DC energy flow, design low self power consumption devices and perform measurements down to every $0.1 \mathrm{~s}$.

III.

NON-EVEN SAMPLING METHOD

Non- even sampling method [6] propose that mains voltage

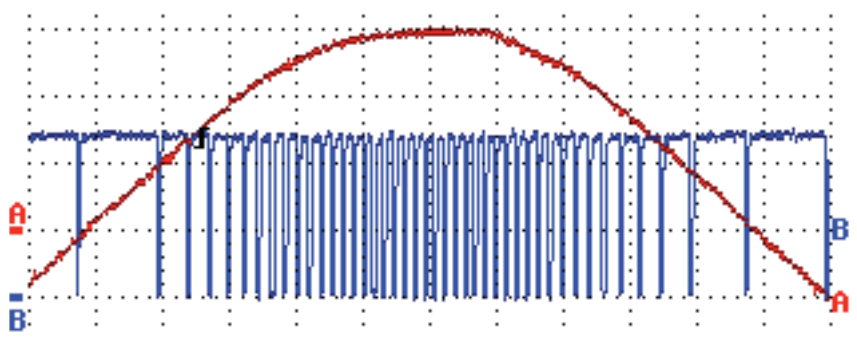

Fig.1.Voltage-frequency converter output signal B v.s. input voltage A.

are converted to frequency by voltage-to-frequency ( $\rightarrow$ f) converter and output frequency are proportional to input voltage (Fig.1.), instead of voltage reading via transformer, voltage divider etc.

Voltage-frequency converter output signal (pulses) are transferred through isolating optocoupler and used as microcontroller's ADC strobe signal. Strobe signal frequency (period) determine current sampling intervals $\Delta \mathrm{t}$.

According to non-even sampling method, amount of consumed or generated electrical energy during the predefined period of time, is directly proportional to the sum of current samples over this time, multiplied by voltage-frequency transfer coefficient, if current sampling rate are modulated by applied voltage value. 


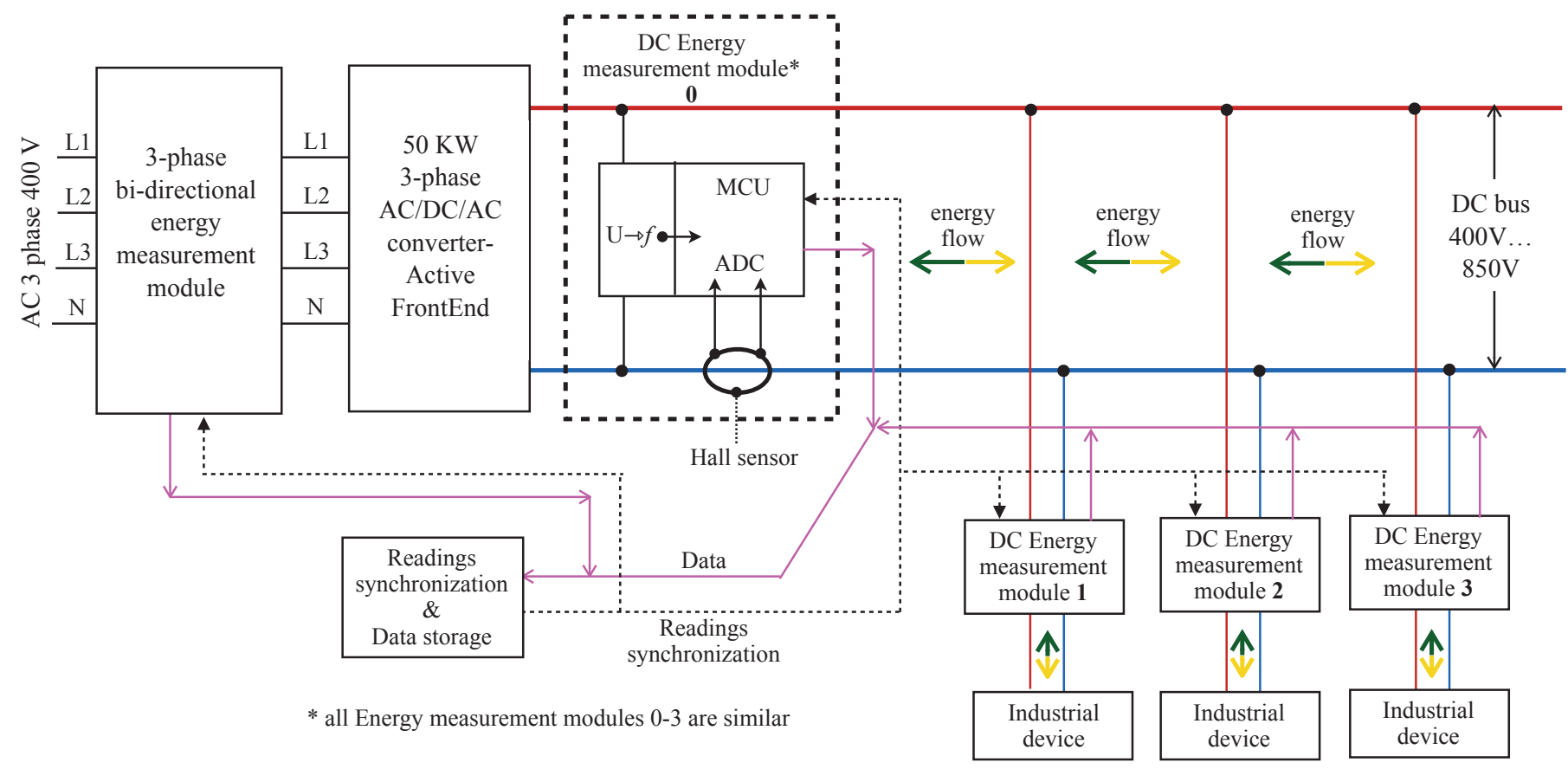

Fig.2. Energy measurement modules installation on AC side and DC micro-grid.

\section{SYNCHRONOUS MEASUREMENT DEVICES INSTALLATION AND DESCRIPTION}

\section{A. Description}

Measurement devices installation diagram on DC microgrid are shown on Fig.2. DC micro-grid is connected to AC mains by active front-and converter.

All DC energy bi-directional flow measurement modules are designed to measure electrical parameter values taking in to account bi-directional energy flow due to recuperation. Overall installed DC micro-grid power is up to $50 \mathrm{KW}$.

AC energy bi-directional flow module practically represent three, similar DC energy flow measurement modules. In this case only one MCU are used for all readings, calculations data storage and communication. Due to bidirectional energy flow, $\mathrm{AC}$ modules utilise two voltage to frequency converters - one for each AC half-period (Fig.3.)

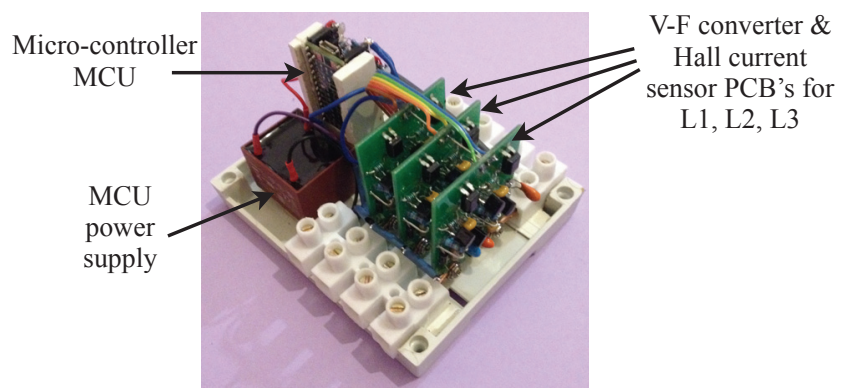

Fig.3. AC 3-phase bidirectional energy consumption measurement module
Module micro-controller unit (MCU) include 32-bit Cortex M4, micro-controller(72 MHz, 12-bit ADC). Popular and very low priced 8-bit, $16 \mathrm{MHz}$ micro controllers with 10-bit ADC can be applied in cases when reduced precision is acceptable mainly form energy flow monitoring and overall estimation.

Data communication are based on UART or USB (preferable) protocols and devices. Industrial systems typically use EtherNet or Profinet protocols. Additional modules or protocol converters must be added in order to realise these protocols.

Energy flow readings from modules can be synchronised via special synchronisation circuit. Such option is necessary if energy values are taken over short time periods - $10 \mathrm{~ms}$ to 500 ms. Short period energy measurement generate important data flow and large data log files.

\section{B. Voltage-frequency convertor}

Designed patent pending voltage - frequency converter is self-powering, electrically isolated from MCU and consume less than $2 \mathrm{~mA}$ if input voltage is $850 \mathrm{~V}$ or less than $1.9 \mathrm{~W}$. Energy self consumption become important is significant number of modules are installed.

Converter input -output curve are shown on Fig.4. Overall tested voltage range (linear frequency change) is $120 \mathrm{~V} \ldots 950 \mathrm{~V}$.

Basically voltage - frequency converter act as native lowpass filter thus eliminating errors caused by noise. 


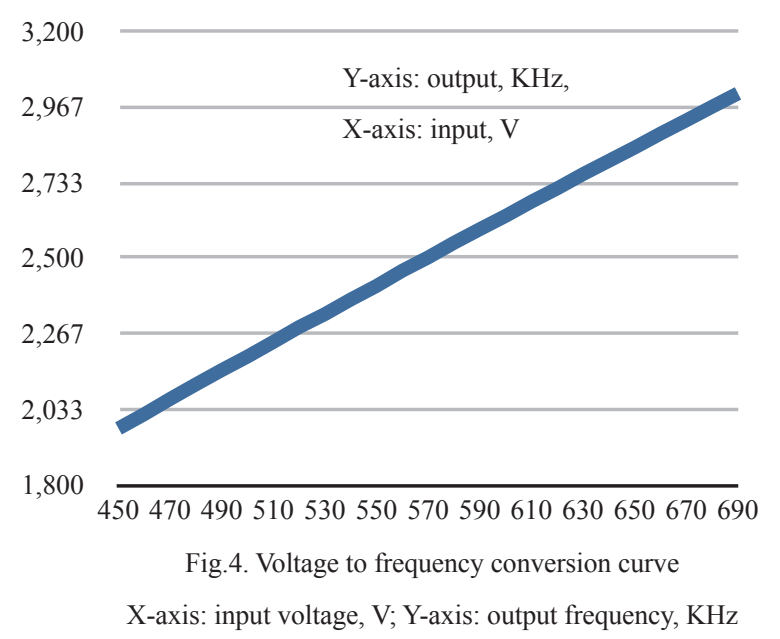

\section{Current sensing}

Today, DC component can be generated and injected in to $\mathrm{AC}$ grids from power electronics devices - solar power, wind power and energy storage converters, smart houses and buildings, etc. Low $\mathrm{DC}$ current values in $\mathrm{AC}$ current are difficult to measure [8].

Described simultaneous energy flow measurement in all 3 phases + current in to neutral allow to detect even small DC injection in to AC grid, possibly take place due to Active FrontEnd converter.

Current measurement are provided by contactless radiometric Hall sensors in all modules.

Sensors patent pending design - absence of ferromagnetic materials in to sensor magnetic circuit but still good resistance to stray magnetics fields - allow to avoid any influence of remanent induction $\mathrm{B}_{\mathrm{r}}$ on magnetisation curve when $\mathrm{H}$ is zero no current flow (Fig.5.).

Exception is initial design where ferrite core was applied (Fig.6., upper left).

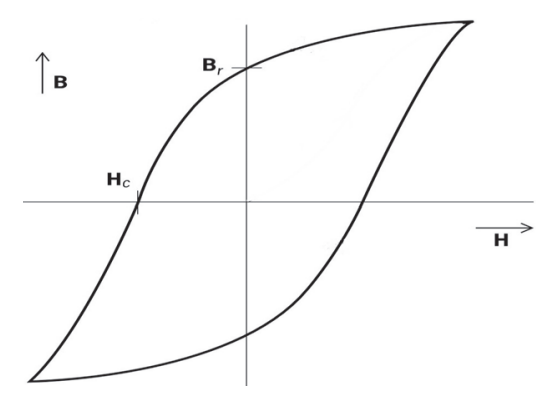

Fig.5. Typical ferromagnetic magnetisation B-H curve

Hall current sensors are able to sense AC and DC current. Current transformers, even with compensation winding, mainly perform the best in to AC environment. Current transformers typically are larger in size too.

Known magneto-resistive magnetic field sensors have some small advantages against Hall sensors but are more expensive and a bit specific implementation.
Ratiometric Hall sensor output voltage $\mathrm{V}_{0}$ typically is a half of sensor supply voltage $\mathrm{V}_{\mathrm{CC}}$ when magnetic field $B$ is zero -no current flows - (1):

$$
V_{0}=\frac{1}{2} V_{c c},
$$

To know real current value, from corresponding to this value output voltage $\mathrm{V}_{0}$ must be subtracted (2):

$$
I \approx V_{R}-V_{0}
$$

Obviously, precise value of $\mathrm{V}_{0}$ is important in order to calculate real current value so periodically $\mathrm{V}_{\mathrm{CC}}$ must be measured to calculate real $\mathrm{V}_{0}$ value. Temperature changes also have influence on $\mathrm{V}_{0}$ value.

Available on the market Hall sensors error under different conditions are in range $0,25 \% \ldots 2.5 \%$. for temperatures around $25^{\circ} \mathrm{C}$. Precision is comparable to current transformer precision on $\mathrm{AC}$ measurements.

$V_{0}$ value or more precisely, "zero current" $V_{R}$ value can be calculated average value from read samples if only AC component must be measured (DC component not exist or isn't important). Well known current transformers also measure only AC component.

In order to calculate $\mathrm{V}_{0} \mathrm{AC}$ voltage symmetry in positive and negative half periods is important due to non-even sampling. Without symmetry there are different number of samples in each half period and $\mathrm{V}_{0}$ can be calculated as (3):

$$
V_{0}=\frac{1}{2}\left(\frac{\sum_{1}^{N 1} i_{+}}{N 1}+\frac{\sum_{1}^{N 2} i_{-}}{N 2}\right),
$$

where

N1-number of samples in positive half period,

N2-number of samples in positive negative period,

i+ particular sample in positive half period,

i. particular sample in positive negative period.

Programmable gain amplifier is applied in front of the ADC in order to perform automatic and MCU controlled measurement range change. Gain can be set as 1, 2, 4 and 8 . Range change doesn't relay on readings average value over time period but on instant sample value and amplifier gain is instantly adjusted for the next sample reading.

Sampling noise reduction are achieved through ADC readings averaging - standard feature for many Cortex M4 micro-controllers. Described designs utilise averaging of 4 readings and ADC sampling time ("ADC ready") is less than 4 microseconds.

\section{Modules design}

Initial design of $\mathrm{AC}$ and $\mathrm{DC}$ energy flow measurement (sensor) modules are shown on Fig.5 Designs take in to account DC micro-grid voltage value, possible DC arcs and average micro-grid power $25-30 \mathrm{KW}$. 


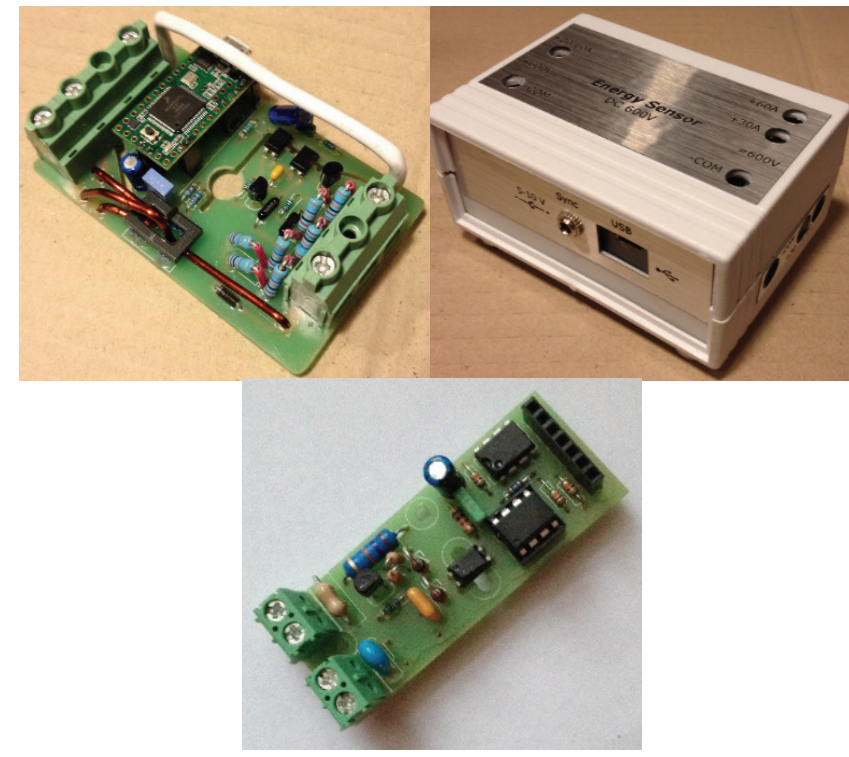

Fig.6. AC (bottom) and DC (top right and left) energy sensor modules

AC modules (Fig.3.,Fig.6. below and Fig.7.) include resettable fuse and varistor protection, DC module include protection against possible reverse DC polarity connection to voltage-frequency converter.

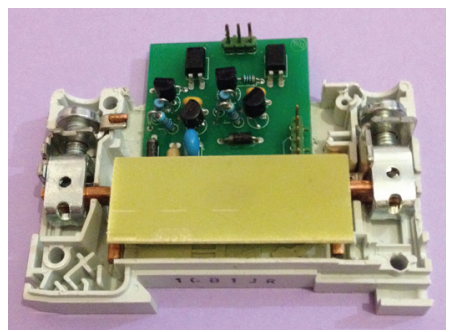

Fig.7. One part of 3-phase 50kW AC energy sensor module

Several developments (based on different MCU) of devices was tested: converters, software and communication.

UART (speed up to 115,2 Kbaud) and USB (speed $12 \mathrm{Mbit} /$ s) communication allow to store readings in to log file or FileMaker Pro database. Communication allow to store measurements starting from $100 \mathrm{~ms}$ intervals.

More fast USB communication cable also provide DC module power supply.

USB communication was choose to lower overall costs, in case if several consumers/generators are in to close distance (up to $5 \mathrm{~m}$ between consumer/generator and USB hub or computer)
UART communication allow more effective implement industrial EtherNet or ProfiNet communication.

\section{IV.}

\section{CONCLUSIONS AND FUTURE WORK}

Synchronised multipoint installation energy flow sensors allow to analyse DC micro-grid parameters, create data log files and store information.

Energy flow sensors also can act as possible electrical equipment fault detectors due to wear-out and ageing - constant increasing of consumed energy turn attention to the possible problems in to future.

Several improvements must be made in to sensors design possibility to install as standard devices in electrical cabinets, for example.

\section{ACKNOWLEDGMENT}

The research leading to these results has received funding from the European Community's seventh framework program under grant agreement No. 609391 (AREUS- Automation and Robotics for European Sustainable manufacturing).

\section{REFERENCES}

1. Atmel AVR1631: Single Phase Energy Meter using XMEGA A, available: http://www.atmel.com/images/doc42039.pdf

2. Apse-Apsitis P., Avotins A., Ribickis L., SYSTEM AND METHOD FOR MONITORING REAL POWER CONSUMPTION, International patent application WO 2013/093554 A1, published 27.06.2013

3. available: http://www.analog.com/static/imported-files/ application notes/AN-679.pdf

4. Apse-Apsitis P., Avotins A., Ribickis L., Zakis.J., Development of Energy Monitoring System for Smart Grid Application, 3rd IFIP WG 5.5/SOSOLNET Doctoral Conference on Computing, Electrical and Industrial Systems, DoCEIS 2012, Costa de Caparica, Portugal, 2012 Proceedings, Springer Heidelberg Dordrecht London New York, ISSN 1868-4238, ISBN 978-3-642-28254-6, p. 347.- 354.

5. Apse-Apsitis P., Avotins A., Ribickis L., Concept of Low-Cost Energy Monitoring System for household Application, Proceedings ELMAR-2011, Zadar, Croatia, ISBN: 978-953-7044-12-1., p.149-152

6. Apse-Apsitis, P. ; Avotins, A. ; Ribickis, L., A different approach to electrical energy consumption monitoring, Power Electronics and Applications (EPE'14-ECCE Europe), 2014, DOI: 10.1109/EPE. 2014.6910970, IEEE CONFERENCE PUBLICATIONS

7. Harmonic Current Emissions, Guidelines to the standard EN 61000-3-2, available: http://www.epsma.org/pdf/PFC\%20Guide November $\% 202010 . p d f$

8. Gertmar, Lars ; Karlsson, P. ; Samuelsson, O., On DC injection to AC grids from distributed generation, Power Electronics and Applications, 2005 European Conference on, 2005, DOI: 10.1109/EPE.2005.219420, IEEE CONFERENCE PUBLICATIONS

This is a post-print of a paper published in Proceedings of the 2015 IEEE 5th International Conference on Power Engineering, Energy and Electrical Drives (POWERENG) [http://dx.doi.org/10.1109/PowerEng.2015.7266362] and is subject to IEEE copyright. 